Ciência Florestal, Santa Maria, v. 28, n. 1, p. 345-356, jan.- mar., 2018

ISSN 1980-5098 @ (1) @ DOI: http://dx.doi.org/10.5902/1980509831605

\title{
POTENCIAL REGENERATIVO DE UMA FLORESTA ECOTONAL NA REGIÃO DO ALTO URUGUAI EM SANTA CATARINA
}

\author{
REGENERATIVE POTENTIAL OF AN ECOTONAL FOREST IN THE UPPER URUGUAY REGION, \\ SANTA CATARINA STATE
}

\author{
Chayane Cristina de Souza ${ }^{1}$ Angélica Dalla Rosa ${ }^{2}$ Karine Souza ${ }^{3}$ Aline Pereira Cruz ${ }^{1}$ \\ Didiane Ana Gonçalves ${ }^{2}$ Francieli Pscheidt ${ }^{3}$, Rodineli Loebens ${ }^{1}$, Pedro Higuchi ${ }^{4}$ Ana Carolina da Silva ${ }^{4}$
}

\section{RESUMO}

Objetivou-se avaliar o potencial regenerativo do componente arbóreo em uma área ecotonal entre Floresta Ombrófila Mista e Estacional Decidual, na região do Alto Uruguai, Santa Catarina. Para isso, foi realizada a caracterização florístico-estrutural do componente arbóreo regenerante em diferentes classes de tamanho e verificada a similaridade florística entre as classes e o componente adulto. Foram consideradas três classes para o componente regenerante: Classe 1, plantas com altura entre 0,2 e $1 \mathrm{~m}$, Classe 2, plantas maiores que $1 \mathrm{~m}$ e até $3 \mathrm{~m}$, e Classe 3 , plantas maiores que $3 \mathrm{~m}$ e com DAP $<5,0 \mathrm{~cm}$. Informações sobre o componente adulto para as mesmas unidades amostrais foram extraídas do banco de dados Laboratório de Dendrologia e Fitossociologia (LABDENDRO), da Universidade do Estado de Santa Catarina. Todas as espécies regenerantes foram classificadas em guildas de regeneração (pioneiras, climácicas exigentes em luz e climácicas tolerantes à sombra), a partir de revisão de literatura e observações de campo. Para todo o componente e para cada classe de tamanho, foram calculados os índices de diversidade de Shannon (H'), de equabilidade de Pielou (J) e os Índices de Regeneração Natural por Classe (RNC) e Total (RNT). As similaridades entre os componentes foram obtidas pelo Índice de Sorensen. Foi realizado um teste de qui-quadrado aplicado a uma tabela de contingência para verificar a existência de associação entre a distribuição dos indivíduos pertencentes a espécies de diferentes guildas de regeneração e a classe de tamanho. Foram amostrados 771 indivíduos regenerantes, distribuídos em 51 espécies, cujos valores de H' e J foram, respectivamente, de 3,06 e 0,77. A espécie com maior RNT foi Nectandra megapotamica (Spreng.) Mez. A similaridade com o componente adulto variou entre 0,46 para a Classe 3 e 0,62 para a Classe 1. Em todas as classes de tamanho ocorreu a predominância de indivíduos de espécies classificadas como clímax exigentes em luz. Conclui-se que as espécies do componente adulto estão representadas, principalmente, na classe de menor tamanho, na qual se encontra a maior parte dos indivíduos amostrados. Palavras-chave: regeneração natural; similaridade florística; sub-bosque.

1 Biólogo, MSc., Departamento de Engenharia Florestal, Universidade do Estado de Santa Catarina, Av. Luiz de Camões, 2090,CEP 88520-000, Lages (SC), Brasil. chayanesouza8@gmail.com / a.line_cruz@yahoo.com.br / rhodineli@hotmail.com

2 Engenheira Florestal, Departamento de Engenharia Florestal, Universidade do Estado de Santa Catarina. Av. Luiz de Camões, 2090, CEP 88520-000, Lages (SC), Brasil. angelica.dalla.rosa@hotmail.com / didi_ana15@hotmail.com

3 Engenheira Florestal, MSc., Departamento de Engenharia Florestal, Universidade do Estado de Santa Catarina, Av. Luiz de Camões, 2090, CEP 88520-000, Lages (SC), Brasil. karisouza@hotmail.com / francieli_pscheidt@hotmail.com

4 Engenheiro Florestal, Dr., Professor do Departamento de Engenharia Florestal, Universidade do Estado de Santa Catarina, Av. Luiz de Camões, 2090, CEP 88520-000, Lages (SC), Brasil. higuchip@gmail.com / carol_sil4@ yahoo.com.br

Recebido para publicação em 14/09/2015 e aceito em 20/10/2016

Ci. Fl., v. 28, n. 1, jan.- mar., 2018 


\begin{abstract}
We aimed to evaluate the regenerative potential of the tree component in an ecotonal area between Araucaria and Deciduous forests, in the Upper Uruguay region, Santa Catarina state. For this, a floristicstructural description of the regenerative component was conducted for different size classes and the floristic similarity among classes and adult component was verified. Three classes were considered for the regenerative component: Class 1, plants from 0.2 up to $1 \mathrm{~m}$ high, Class 2, plants greater than $1 \mathrm{~m}$ up to $3 \mathrm{~m}$ high, and Class 3, plants greater than $3 \mathrm{~m}$ high, with cbh $<5.0 \mathrm{~cm}$. Information about the adult component for the same sampling units were extract from the database of the Dendrology and Phytosociology Laboratory (LABDENRO), of Santa Catarina State University. All the regenerative species were classified into regeneration guilds (pioneers, light demanding climax, shade tolerant climax), based on literature review and on field observations. For the whole component and for each size class, the Shannon diversity (H') and Pielou Eveness (J) indexes, and the Class (RNC) and Total (RNT) Natural Regeneration Indexes were determined. The floristic similarities between components were obtained by Sorensen Index. A chi-square applied to a contingency table was conducted to verify the existence of association between the distribution of individuals belonging to different regeneration guilds and size classes. A total of 771 regenerative individuals were sampled, belonging to 52 species, whose H' and J were, respectively, 3.06 and 0.77 . The species with the greatest RNT was Nectandra megapotamica (Spreng.) Mez. The similarity with the adult component varied between 0.46 for Class 3 and 0.62 for Class 1 . In all size classes, there was the predominance of individuals of climax light demanding species. We conclude that the adult species component was mostly represented within the smaller plant size class, where the largest proportion of sampled individuals was found.
\end{abstract}

Keywords: natural regeneration; floristic similarity; understory.

\title{
INTRODUÇÃO
}

O estabelecimento e o desenvolvimento de indivíduos arbóreos jovens em condição de sub-bosque, processo denominado como regeneração natural, representa uma importante etapa na dinâmica de florestas, pois é quando ocorrem as maiores pressões seletivas (POORTER, 2007). Assim, durante o processo, são definidas as espécies com o potencial de ocuparem os diferentes estratos do perfil vertical da floresta (FELFILI et al., 2000; SALLES; SCHIAVINI, 2007).

Entre a germinação do propágulo até a idade adulta, uma árvore passa por uma série de filtros ecológicos abióticos e bióticos, além de se sujeitar a vários distúrbios ambientais estocásticos (e.g., formações de clareiras). Por isso, espera-se que apenas um pequeno percentual de indivíduos arbóreos que se estabelecem inicialmente consiga completar todo o ciclo de vida em uma floresta natural. Desta forma, a relação florística entre o componente adulto e regenerativo em uma floresta depende de vários fatores, como aqueles relacionados à autoecologia das espécies, condições ambientais e distúrbios, de forma que esta pode apresentar diferenças em função do estágio sucessional florestal (ASSIS; WITTMAN, 2011).

Dentre os fatores abióticos, destacam-se aqueles associados às exigências ecológicas das espécies, como as condições microclimáticas (e.g. luz) (KOBE, 1999) e o ambiente edáfico (e.g. propriedades físico-químicas, profundidade, relevo) (PALMIOTTO et al., 2004; HIGUCHI et al., 2015). Além disso, vários autores também têm destacado a importância das relações bióticas, associadas à riqueza e à densidade do componente adulto (HIGUCHI et al., 2015), relações ecológicas inter (SANTOS et al., 2015) e intraespecífica (JANZEN, 1970; WEBB; GILBERT; DONOGHUE, 2006), associações simbióticas obrigatórias (JOHNSON; GRAHAM; SMITH, 1997) e dispersão limitada (INMAN-NARAHARI et al., 2013). Em função das diferenças ecológicas das espécies, as mesmas podem ser classificadas em grupos funcionais distintos. Por exemplo, Swaine e Whitmore (1988) classificaram as espécies em função de suas exigências lumínicas em pioneiras, sendo aquelas dependentes de elevada incidência de luz para germinar e completar todo o ciclo de vida, e climácicas, que toleram diferentes níveis de sombreamento.

Neste sentido, conhecer a fitossociologia das espécies arbóreas jovens em condições de sub-bosque

Ci. Fl., v. 28, n. 1, jan.- mar., 2018 
permite realizar inferências sobre o potencial regenerativo de uma floresta em condições naturais (GAMA; BOTELHO; BENTES-GAMA, 2002; SILVA et al., 2007), sendo fundamental para a definição de estratégias de conservação e manejo sustentável. Assim, considerando o exposto, o presente trabalho teve como principal objetivo avaliar o potencial regenerativo de uma floresta em área ecotonal entre Floresta Estacional Decidual (FED) e Floresta Ombrófila Mista (FOM), na região do Alto Uruguai, em Santa Catarina. Para isto, foi realizada a caracterização florístico-estrutural do componente regenerativo em diferentes classes de tamanho, sendo verificada a similaridade florística entre as classes e o componente adulto.

\section{MATERIAL E MÉTODOS}

A área estudada é um fragmento que se encontra em um ecótono entre as formações de Floresta Ombrófila Mista e Estacional Decidual (classificação do IBGE, 2012), localizado em um tributário do rio

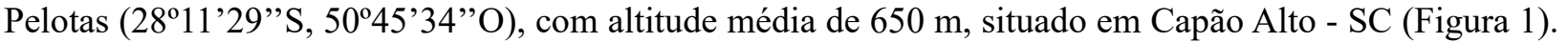
O clima é Cfb, segundo Köppen, e a precipitação no ano, com boa distribuição, varia entre 1.200 a 1.900 $\mathrm{mm}$. A topografia varia entre suave-ondulada a ondulada. O fragmento se encontra nas margens da represa da hidrelétrica de Barra Grande, que teve a formação do lago no ano de 2005. Conforme histórico da área obtido a partir de entrevista com o proprietário, este fragmento, atualmente, está protegido e se encontra em avançado estágio sucessional.

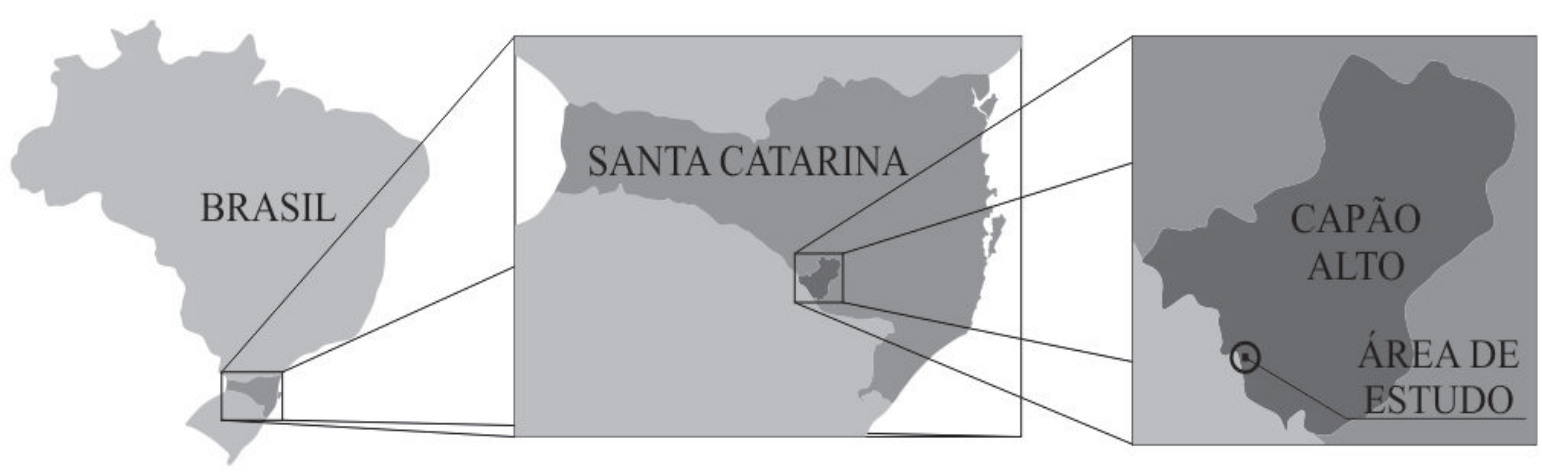

FIGURA 1: Localização geográfica do remanescente estudado de floresta de ecótono na região do Alto Uruguai em Santa Catarina.

FIGURE 1: Geographical location of study ecotonal forest remnant in the Upper Uruguay region, SC state.

Para o levantamento vegetacional, foram instaladas 30 unidades amostrais, com área diferenciada segundo a classe de tamanho do regenerante. As unidades amostrais foram distribuídas de forma estratificada-sistemática em uma área de encosta, na margem do reservatório. Assim, foram considerados os estratos inferior (próximo à margem do reservatório e de topografia mais plana), intermediário (no meio da encosta e de topografia declivosa) e superior (no topo da encosta, de topografia declivosa e com a presença de clareiras com diferentes tamanhos e tempo de formação). Foram alocadas 10 unidades amostrais por estrato, distanciadas $10 \mathrm{~m}$ entre si.

Informações sobre o componente arbóreo adulto (diâmetro a altura do peito - DAP $\geq 5,0 \mathrm{~cm}$, amostrado por Souza et al. (2015)), referente à abundância e à identificação de cada espécie nas 30 unidades amostrais (cada uma de $10 \times 20 \mathrm{~m}$ ), foram extraídas do banco de dados do LABDENDRO da Universidade do Estado de Santa Catarina. O componente arbóreo regenerante (DAP $<5,0 \mathrm{~cm}$ ) foi amostrado em subunidades amostrais alocadas no interior das unidades amostrais dos adultos, com tamanho diferenciado de acordo com a classe de tamanho da planta, segundo metodologia modificada de Volpato (1994): Classe 1, plantas de $20 \mathrm{~cm}$ a $1 \mathrm{~m}$ de altura, amostradas em parcelas de $5 \mathrm{~m}^{2}(1 \times 5 \mathrm{~m})$; Classe 2, plantas maiores do que $1 \mathrm{~m}$ e até $3 \mathrm{~m}$ de altura, inventariadas em parcelas de $10 \mathrm{~m}^{2}(1 \times 10 \mathrm{~m})$; e Classe 3 , plantas acima de $3 \mathrm{~m}$ de altura e menores do que 5,0 cm de DAP, contabilizadas em parcelas de $20 \mathrm{~m}^{2}(1 \times 20 \mathrm{~m})$. No interior das subunidades amostrais, todos os indivíduos arbóreos foram etiquetados, identificados e determinadas suas 
classes de altura. Os que não tiveram sua identidade determinada, foram obtidas coletas e as identificações foram realizadas por especialista ou bibliografia especializada. As espécies foram organizadas em famílias segundo o APG IV (ANGIOSPERM PHYLOGENY GROUP, 2016).

Para o total de indivíduos regenerantes e para cada classe separadamente, foi quantificada a densidade absoluta, riqueza de espécies, riqueza padronizada pela rarefação, índice de Shannon (H') e equabilidade de Pielou (J). A suficiência amostral do componente regenerativo foi determinada utilizandose a curva de acumulação de espécies, pelo método da aleatorização, com 1.000 permutações. Foram estimadas as densidades e frequências absolutas e relativas de cada espécie amostrada na regeneração natural. Por meio da média dos valores de frequência relativa e densidade relativa, foram calculados os Índices de Regeneração Natural por Classe (RNC) e Total (RNT) (VOLPATO, 1994). A similaridade entre as diferentes classes de tamanho, representando as fases ontogenéticas do componente arbóreo (classes de tamanho da regeneração natural, junto com o componente adulto), foi obtida pelo Índice de Sorensen, utilizando uma matriz de presença e ausência.

Todos os indivíduos amostrados foram classificados de acordo com a guilda de regeneração da espécie à qual pertence, em pioneiras, clímax tolerante à sombra ou clímax exigente em luz, de acordo com a classificação de Swaine e Whitmore (1988), adaptada por Oliveira Filho et al. (1994). Esta classificação das espécies baseou-se em observações realizadas em campo e com base na literatura (LOREGIAN et al., 2012; SOUZA et al., 2015). Foi realizado um teste de qui-quadrado aplicado a uma tabela de contingência para verificar se existe relação entre a distribuição de indivíduos em diferentes guildas de regeneração e classes de tamanho. Utilizou-se a linguagem de programação estatística R (R DEVELOPMENT CORE TEAM, 2015), junto com o pacote Vegan (OKSANEN et al., 2015), assim como planilha eletrônica, para analisar os dados.

\section{RESULTADOS E DISCUSSÃO}

A densidade absoluta dos indivíduos arbóreos regenerantes foi de 40.500 ind.ha $^{-1}$ (Tabela 1). Da menor (Classe 1) para a maior classe (Classe 3) de tamanho, ocorreu uma acentuada redução na densidade de plantas, na ordem de $96,5 \%$. Esta redução da densidade em função do tamanho de plantas não se repetiu para a riqueza padronizada, diversidade e equabilidade, cujos valores para toda comunidade foram, respectivamente, 51 espécies, 3,06 e 0,77. Os maiores valores de riqueza padronizada e diversidade foram observados para a classe de tamanho intermediária (Classe 2) e a maior equabilidade foi observada para a Classe 3.

TABELA 1: Densidade, riqueza e índices de diversidade das espécies arbóreas, por classe de altura, em um remanescente de floresta de ecótono na região do Alto Uruguai, Santa Catarina.

TABLE 1: Density, richness and diversity indexes of tree species, by height class, in an ecotonal forest remnant in the Upper Uruguay region, SC state.

\begin{tabular}{cccccc}
\hline & Densidade absoluta (ind./ha) & Riqueza & Riqueza padronizada $^{1}$ & Shannon & Pielou \\
\hline Classe 1 & 31.800 & 41 & 20 & 2,80 & 0,75 \\
Classe 2 & 7.600 & 39 & 25 & 3,14 & 0,85 \\
Classe 3 & 1.100 & 21 & 21 & 2,70 & 0,88 \\
Total & 40.500 & 51 & - & 3,06 & 0,77 \\
\hline
\end{tabular}

Em que: ${ }^{1}$ Riqueza calculada pela rarefação.

A elevada densidade na classe de menor tamanho de regenerantes é típica de florestas inequiâneas e sugere que o banco de plântulas e de indivíduos mais jovens representa um importante componente do processo de regeneração florestal (SCHORN; GALVÃO, 2006). De acordo com Sccoti et al. (2011), este padrão indica uma grande produção de propágulos, permitindo que indivíduos ingressem no banco de plântulas, mas com poucos, de fato, conseguindo atingir tamanhos maiores. Isto corre, pois, na fase inicial de estabelecimento, as plantas encontram-se no período de maior vulnerabilidade de seus ciclos de vida (POORTER, 2007), de forma que a redução do tamanho populacional observada nas classes de maiores 
tamanhos é esperada. Durante a fase de estabelecimento, os propágulos, ao chegarem ao solo florestal, ficam sujeitos a filtros ecológicos, abióticos (e.g., stress ambientais) e bióticos (e.g., competição inter e intraespecífica, herbivoria, presença de organismos simbióticos) (JANZEN, 1970; JOHNSON; GRAHAM; SMITH, 1997; GEORGE; BAZZAZ, 1999; WEBB; GILBERT; DONOGHUE, 2006; LARPKERN; MOE; TOTLAND, 2011). Destaca-se, ainda, que a maior dominância ecológica na Classe 1, refletida pelo valor do Índice de Equabilidade, associada à elevada densidade, seguida por uma mudança de padrão nas maiores classes de tamanho, caracterizado por maior equabilidade e menor densidade de plantas, indica que os filtros ecológicos representam uma limitação para o tamanho populacional, mas não para o número de espécies.

Em relação ao perfil florístico, foram identificadas 51 espécies (Tabela 2). A suficiência amostral foi satisfatória para quantificação da riqueza da área, pois houve tendência à estabilização da curva de acumulação de espécies (Figura 2), uma vez que com a inserção das três últimas unidades amostrais, que representam $10 \%$ da área amostral, obteve-se o aumento em riqueza de apenas 1,4\%. Porém, a estabilização de uma curva ocorre, de fato, em seu ponto de inflexão (SCHILLING; BATISTA, 2008), cujo o valor do coeficiente angular da tangente é zero, indicando que não há incremento no número de espécies. Neste caso, o valor obtido para o coeficiente angular da tangente no ponto da curva representado pela penúltima parcela, foi 0,56 . Isto demonstra a necessidade de inclusão de pelo menos duas parcelas para a amostragem de uma nova espécie, o que indica a adequabilidade do esforço amostral para a caracterização da composição florística da área.

TABELA 2: $\quad$ Espécies amostradas e Índice de Regeneração Natural por Classe (RNC, em \%) e Total (RNT, em \%), e guilda de regeneração $(\mathrm{Gr})$, em um remanescente de floresta de ecótono na região do Alto Uruguai em Santa Catarina.

TABLE 2: $\quad$ Sampled species and Natural Regeneration Index by Class (RNC, in \%) and Total (RNT, in \%), and regeneration guild $(\mathrm{Gr})$, in an ecotonal forest remnant in the Upper Uruguay region, SC state.

\begin{tabular}{|c|c|c|c|c|c|}
\hline Famílias/espécies & $\mathrm{RNC}_{1}$ & $\mathrm{RNC}_{2}$ & $\mathrm{RNC}_{3}$ & RNT & Gr \\
\hline \multicolumn{6}{|l|}{ ANNONACEAE } \\
\hline Annona neosalicifolia H.Rainer & 0,21 & 0,21 & 0,00 & 0,42 & CEL \\
\hline Annona rugulosa (Schltdl.) H.Rainer & 0,80 & 0,51 & 0,00 & 1,31 & CEL \\
\hline \multicolumn{6}{|l|}{ APOCYNACEAE } \\
\hline Aspidosperma australe Müll.Arg. & 0,21 & 0,00 & 0,00 & 0,21 & CEL \\
\hline \multicolumn{6}{|l|}{ ARALIACEAE } \\
\hline Oreopanax fulvus Marchal & 0,00 & 0,00 & 0,15 & 0,15 & CEL \\
\hline \multicolumn{6}{|l|}{ ARAUCARIACEAE } \\
\hline Araucaria angustifolia (Bertol.) Kuntze & 0,21 & 0,34 & 0,00 & 0,55 & CEL \\
\hline \multicolumn{6}{|l|}{ ASTERACEAE } \\
\hline Moquiniastrum polymorphum (Less.) G. Sancho & 0,46 & 0,34 & 0,00 & 0,80 & $\mathrm{P}$ \\
\hline \multicolumn{6}{|l|}{ CARDIOPTERIDACEAE } \\
\hline Citronella gongonha (Mart.) R.A.Howard & 0,21 & 0,00 & 0,00 & 0,21 & CEL \\
\hline \multicolumn{6}{|l|}{ CELASTRACEAE } \\
\hline Maytenus aquifolia Mart. & 0,72 & 0,25 & 0,00 & 0,97 & CTS \\
\hline Schaefferia argentinensis Speg. & 0,80 & 0,68 & 0,15 & 1,63 & CEL \\
\hline \multicolumn{6}{|l|}{ EUPHORBIACEAE } \\
\hline Gymnanthes klotzschiana Müll.Arg. & 0,21 & 0,34 & 0,15 & 0,70 & CEL \\
\hline Sebastiania brasiliensis Spreng. & 2,01 & 0,84 & 0,60 & 3,45 & CTS \\
\hline \multicolumn{6}{|l|}{ FABACEAE } \\
\hline Dalbergia frutescens (Vell.) Britton & 0,84 & 0,17 & 0,00 & 1,01 & CEL \\
\hline Machaerium paraguariense Hassl. & 0,72 & 0,17 & 0,60 & 1,48 & CEL \\
\hline
\end{tabular}


TABELA 2: Continuação...

TABLE 2: Continued...

\begin{tabular}{|c|c|c|c|c|c|}
\hline Famílias/espécies & $\mathrm{RNC}_{1}$ & $\mathrm{RNC}_{2}$ & $\mathrm{RNC}_{3}$ & RNT & Gr \\
\hline Muellera campestris (Mart. ex Benth.) M.J. Silva \& A.M.G. Azevedo & 0,72 & 0,38 & 1,37 & 2,46 & CEL \\
\hline Myrocarpus frondosus Allemão & 0,29 & 0,00 & 0,15 & 0,44 & CEL \\
\hline Parapiptadenia rigida (Benth.) Brenan & 2,59 & 1,61 & 0,00 & 4,21 & CEL \\
\hline INDERTEMINADA & 0,21 & 0,00 & 0,00 & 0,21 & $*$ \\
\hline \multicolumn{6}{|l|}{ LAURACEAE } \\
\hline Nectandra megapotamica (Spreng.) Mez & 12,08 & 1,06 & 0,75 & 13,88 & CEL \\
\hline Ocotea puberula (Rich.) Nees & 2,00 & 0,55 & 0,00 & 2,54 & CEL \\
\hline Ocotea pulchella Mart. & 0,63 & 0,00 & 0,00 & 0,63 & CEL \\
\hline Ocotea sp. 1 & 0,21 & 0,17 & 0,00 & 0,38 & $*$ \\
\hline Ocotea sp. 2 & 0,21 & 0,00 & 0,00 & 0,21 & $*$ \\
\hline \multicolumn{6}{|l|}{ MELIACEAE } \\
\hline Cedrela fissilis Vell. & 0,00 & 0,17 & 0,13 & 0,30 & CEL \\
\hline Trichilia elegans A.Juss. & 4,13 & 2,79 & 0,30 & 7,22 & CEL \\
\hline \multicolumn{6}{|l|}{ MYRTACEAE } \\
\hline Blepharocalyx salicifolius (Kunth) O.Berg & 1,06 & 0,76 & 0,00 & 1,82 & CEL \\
\hline Calyptranthes concinna DC. & 0,21 & 0,00 & 0,00 & 0,21 & CTS \\
\hline Campomanesia xanthocarpa O.Berg & 2,56 & 1,90 & 1,37 & 5,83 & CEL \\
\hline Eugenia uniflora $\mathrm{L}$ & 2,02 & 2,36 & 0,96 & 5,34 & CEL \\
\hline Eugenia uruguayensis Cambess. & 0,42 & 0,17 & 0,00 & 0,59 & CTS \\
\hline Myrcengenia mesomischa (Burret) D.Legrand & 0,00 & 0,17 & 0,00 & 0,17 & CTS \\
\hline Myrcianthes gigantea (D.Legrand) D.Legrand & 0,84 & 0,34 & 0,30 & 1,48 & CEL \\
\hline Myrcianthes pungens (O.Berg) D.Legrand & 0,42 & 0,00 & 0,15 & 0,57 & CEL \\
\hline \multicolumn{6}{|l|}{ OLEACEAE } \\
\hline Ligustrum lucidum W.T.Ait. & 0,00 & 0,51 & 0,00 & 0,51 & CEL \\
\hline \multicolumn{6}{|l|}{ PICRAMNIACEAE } \\
\hline Picramnia parvifolia Engl. & 0,21 & 0,00 & 0,00 & 0,21 & CEL \\
\hline \multicolumn{6}{|l|}{ PODOCARPACEAE } \\
\hline Podocarpus lambertii Klotzsch ex Endl. & 0,00 & 0,00 & 0,15 & 0,15 & CEL \\
\hline \multicolumn{6}{|l|}{ PRIMULACEAE } \\
\hline Myrsine coriacea (Sw.) Roem. \& Schult. & 0,63 & 0,17 & 0,00 & 0,80 & CEL \\
\hline Myrsine lorentziana (Mez) Arechav. & 0,21 & 0,00 & 0,00 & 0,21 & $\mathrm{P}$ \\
\hline \multicolumn{6}{|l|}{ RHAMNACEAE } \\
\hline Scutia buxifolia Reissek & 0,00 & 0,17 & 0,00 & 0,17 & CEL \\
\hline \multicolumn{6}{|l|}{ RUBIACEAE } \\
\hline Coutarea hexandra (Jacq.) K.Schum. & 0,79 & 0,84 & 0,00 & 1,63 & CTS \\
\hline Rudgea parquioides (Cham.) Müll. Arg. & 0,00 & 0,17 & 0,00 & 0,17 & CEL \\
\hline \multicolumn{6}{|l|}{ RUTACEAE } \\
\hline Pilocarpus pennatifolius Lem. & 0,96 & 0,55 & 0,00 & 1,51 & CTS \\
\hline \multicolumn{6}{|l|}{ SALICACEAE } \\
\hline Banara tomentosa Clos & 0,38 & 0,85 & 0,15 & 1,38 & CTS \\
\hline
\end{tabular}

Continua... 
TABELA 2: Continuação...

TABLE 2: Continued...

\begin{tabular}{lccccc}
\hline Famílias/espécies & $\mathrm{RNC}_{1}$ & $\mathrm{RNC}_{2}$ & $\mathrm{RNC}_{3}$ & $\mathrm{RNT}$ & $\mathrm{Gr}$ \\
\hline Casearia decandra Jacq. & 0,43 & 0,17 & 0,30 & 0,90 & $\mathrm{CTS}$ \\
\hline SAPINDACEAE & & & & & \\
Allophylus edulis (A.St.-Hil., Cambess. \& A.Juss.) Radlk. & 7,43 & 2,61 & 0,30 & 10,34 & $\mathrm{CEL}$ \\
Allophylus guaraniticus (A.St.-Hil.) Radlk. & 3,77 & 1,74 & 0,15 & 5,65 & $\mathrm{CTS}$ \\
Cupania vernalis Cambess. & 6,64 & 1,53 & 0,30 & 8,46 & $\mathrm{CEL}$ \\
Matayba elaeagnoides Radlk. & 2,30 & 0,68 & 0,45 & 3,43 & $\mathrm{CEL}$ \\
\hline SOLANACEAE & & & & & \\
Brunfelsia cuneifolia J.A.Schmidt & 0,00 & 0,51 & 0,32 & 0,83 & $\mathrm{CTS}$ \\
Cestrum intermedium Sendtn. & 0,21 & 0,00 & 0,00 & 0,21 & P \\
Solanum pseudoquina A.St.-Hil. & 0,00 & 0,21 & 0,00 & 0,21 & $\mathrm{CEL}$ \\
\hline STYRACACEAE & & & & & \\
Styrax leprosus Hook. \& Arn. & 0,88 & 0,76 & 0,00 & 1,64 & $\mathrm{CEL}$ \\
\hline VERBENACEAE & & & & & \\
Duranta vestita Cham. & 0,00 & 0,17 & 0,00 & 0,17 & P \\
\hline TOTAIS & 62,84 & 27,94 & 9,22 & 100,00 &
\end{tabular}

Em que: $\mathrm{C}_{1}$ : Classe 1, $\mathrm{C}_{2}$ : Classe 2, $\mathrm{C}_{3}$ : Classe 3, CEL: clímax exigente em luz, CTS: clímax tolerante à sombra e P: pioneira.

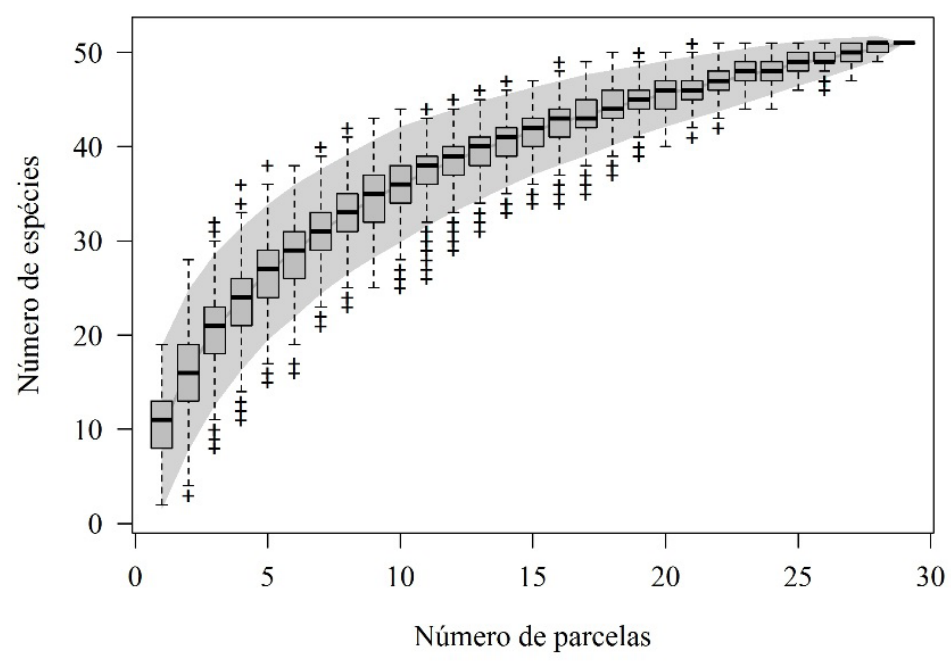

FIGURA2: Curva de acumulação das espécies do componente regenerativo amostradas em um remanescente de floresta em ecótono na região do Alto Uruguai em Santa Catarina.

FIGURE 2: Accumulation curve of regenerative component species sampled in an ecotonal forest remnant in the Upper Uruguay region, SC state.

Considerando as espécies com maior importância relativa, observa-se o caráter ecotonal da área, uma vez que os táxons que se destacaram têm sido relatados como relevantes para a regeneração natural tanto em área de FOM, quanto em FED, no Sul do Brasil. Como observado no Inventário Florístico Florestal de Santa Catarina (VIBRANS et al., 2012a; 2012b), Casearia decandra Jacq., Matayba elaeagnoides Radlk. e Gymnanthes klotzschiana Müll.Arg se destacam no componente regenerativo em áreas de FOM; e 
Muellera campestris (Mart. ex Benth.) M.J. Silva \& A.M.G. Azevedo, Trichilia elegans A.Juss. e Nectandra megapotamica em áreas de FED. Já Cupania vernalis Cambess. foi relatada pelos mesmos autores como uma espécie importante no componente regenerativo de ambas fitofisionomias. Destaca-se que o elemento mais representativo da FOM, Araucaria angustifolia (Bertol.) Kuntze, esteve presente com baixa representatividade na regeneração natural, uma vez que o ambiente avaliado, representado por condições de sub-bosque e baixa altitude relativa, pode ser considerado marginal para o seu estabelecimento (SOUZA, 2007; HIGUCHI et al., 2013a). Esta variação das espécies mais relevantes do componente regenerativo entre diferentes fitofisionomias reflete um padrão fitogeográfico já observado para o componente adulto, relacionado à influência de aspectos ecológicos e históricos, associados, principalmente, ao clima e rotas de migração de espécies arbóreas (HIGUCHI et al., 2013b).

Destaca-se, ainda, a presença de Ligustrum lucidum W. T. Aiton, espécie exótica considerada invasora, também encontrada em outras florestas naturais do sul do Brasil, tanto em FED (e.g. HUMMEL et al., 2014) como em FOM (e.g. GUIDINI et al., 2014). L. lucidum tem a capacidade de substituir o estrato médio de árvores em florestas, além de ter abundante produção de sementes e dispersão zoocórica, apresentando alta taxa de crescimento e de sobrevivência em relação às espécies nativas (ARAGÓN; GROOM, 2003).

Dentre as 15 espécies com maior RNT (Figura 3), 10 apresentaram indivíduos nas três classes de tamanho, o que indica que as mesmas se encontram bem-adaptadas às condições de sub-bosque e que, teoricamente, possuem maior potencial de estabelecimento na floresta (CITADINI-ZANETTE, 1995; SILVA et al., 2007). Nectandra megapotamica se destacou pelo elevado valor de RNC na Classe 1, porém, com baixa representatividade nas outras classes de tamanho. Este padrão sugere que esta espécie tem elevada produção de propágulos, no entanto, baixa capacidade competitiva ou de estabelecimento. Na Classe 2 se destacaram Allophylus edulis (A.St.-Hil., Cambess. \& A.Juss.) Radlk. e Trichilia elegans, e na Classe 3 se destacaram Campomanesia xanthocarpa O.Berg e Muellera campestris. Ressalta-se que a capacidade de uma espécie se manter suprimida ou apresentar um crescimento mesmo que lento em condição de subbosque, alcançando, assim, estágios ontogenéticos posteriores, representa uma das principais vantagens

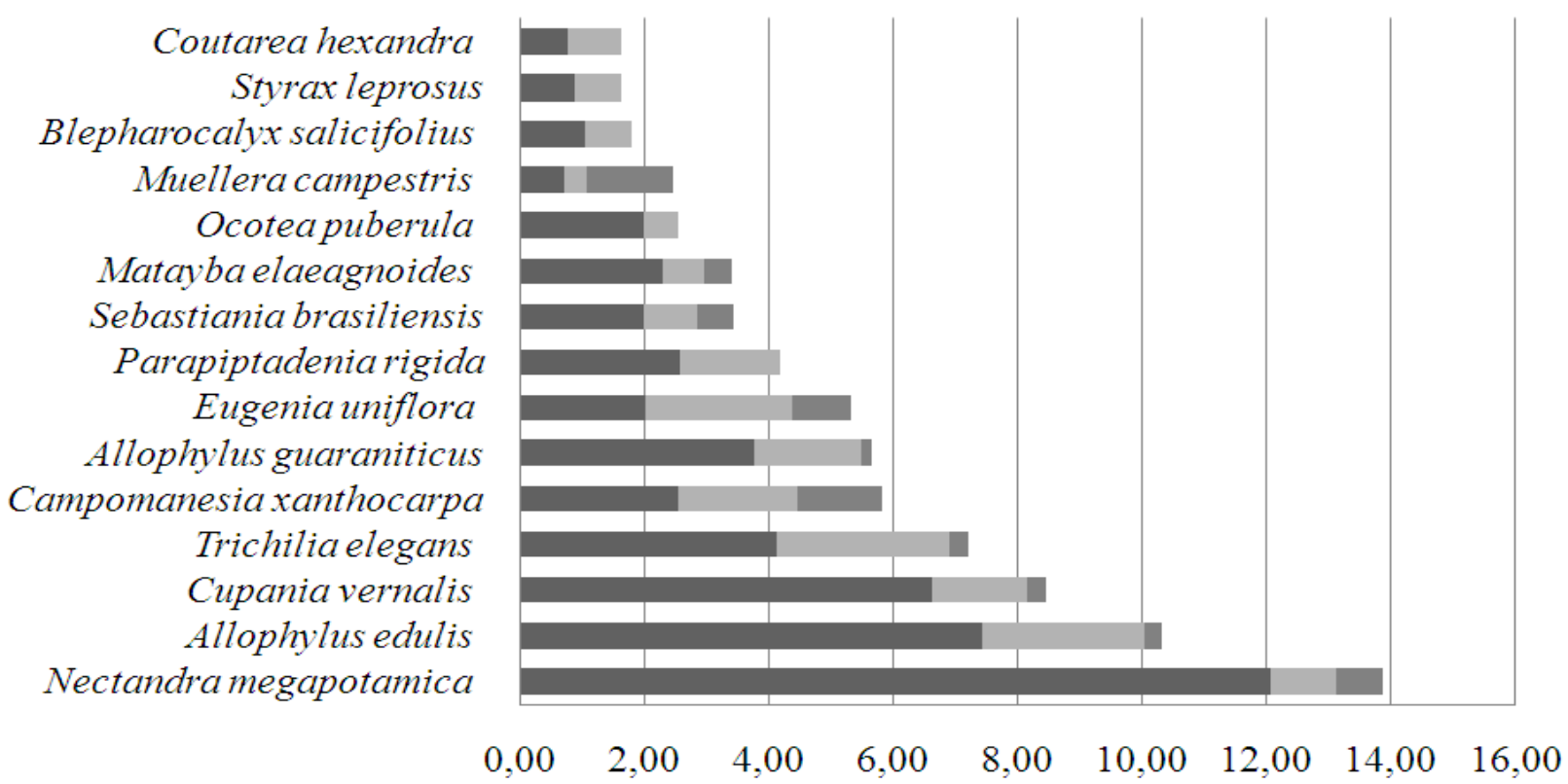

FIGURA 3: Índice de Regeneração Natural em cada classe (C1, C2 e C3), das 15 espécies de maior Índice de Regeneração Natural Total em um remanescente de floresta ecotonal na região do Alto Uruguai em Santa Catarina.

FIGURE 3: Natural Regeneration Index by Class (C1, C2 and $\mathrm{C} 3$ ) of the 15 species most Natural Regeneration Index by Total in an ecotonal forest remnant in the Upper Uruguay region, SC state. 
A similaridade dos regenerantes com o componente adulto variou entre 0,46 para a Classe 3 até 0,62 para a Classe 1 (Tabela 3). Assis e Wittmann (2011), a partir de uma revisão de trabalhos realizados na Amazônia, verificaram que a similaridade entre as espécies que ocupam o dossel e o sub-bosque pode variar em função do estágio sucessional. Assim, a semelhança entre os adultos e as diferentes classes de tamanho pode estar refletindo este aspecto sucessional, no presente caso, predominantemente tardio, além das estratégias ecológicas das espécies analisadas e a influência de filtros ecológicos. De forma geral, os resultados indicam que as espécies do componente adulto estão mais bem representadas na classe de menor tamanho, do que nas maiores classes da regeneração natural, demonstrando que boa parte consegue se estabelecer inicialmente, mas poucas conseguem de fato se desenvolver.

Tendo em vista que as espécies arbóreas apresentam diferentes exigências quanto ao ambiente lumínico, seria natural supor que o padrão observado fosse explicado também por este aspecto. Seguindo este raciocínio, na Classe 1 existiria a predominância de espécies classificadas como clímax exigentes em luz, que se regeneram, mas não apresentam capacidade de avançar para as classes de maior tamanho em condições de sub-bosque; e na Classe 3 existiria o predomínio de espécies clímax tolerantes ao sombreamento, típicas de sub-bosque, que não chegam alcançar o dossel. Porém, este não foi o caso, uma vez que a distribuição dos indivíduos nas diferentes classes de tamanho não apresentou relação com as diferentes guildas de regeneração (Tabela 4) (qui-quadrado $=5,67 ; p=0,225$ ), de forma que os indivíduos pertencentes às espécies clímax exigentes em luz predominaram ao longo de todo perfil vertical da floresta. Assim, fica evidenciado o particionamento do perfil vertical da floresta por espécies que apresentam diferentes estratégias de vida, porém, não associadas à guilda de regeneração. Este resultado indica que o processo de regeneração é um processo complexo, dependente de vários fatore ecológicos, além das condições lumínicas.

TABELA 3: Similaridade entre estratos do componente regenerante (classes 1,2 e 3) e adulto por meio do Índice de Sorensen, em um remanescente de floresta de ecótono na região do Alto Uruguai em Santa Catarina.

TABLE 3: Similarity between strata of regenerating component (classes 1, 2 and 3) and adult through Sorensen index in an ecotonal forest remnant in the Upper Uruguay region, SC state.

\begin{tabular}{lccc}
\hline & Adultos & Classe 3 & Classe 2 \\
\hline Classe 3 & 0,46 & & \\
Classe 2 & 0,57 & 0,57 & \\
Classe 1 & 0,62 & 0,59 & 0,78 \\
\hline
\end{tabular}

TABELA 4: Número de indivíduos por classe e por guildas de regeneração (CTS = clímax tolerantes à sombra; $\mathrm{CEL}=$ clímax exigente em luz; $\mathrm{P}$ = pioneira) em um remanescente de floresta de ecótono na região do Alto Uruguai em Santa Catarina. Valores entre parênteses representam o esperado para uma distribuição uniforme de acordo com o teste de qui-quadrado (qui-quadrado $=5,67 ; p=0,225$ ).

TABLE 4: Number of individuals by class and by regeneration guilds $(\mathrm{CTS}=$ climax tolerant shadow; $\mathrm{CEL}=$ climax demanding in light; $\mathrm{P}=$ pioneer $)$ in an ecotonal forest remnant in the Upper Uruguay region, SC state. Values in brackets represent the expected for a uniform distribution according to the chi-square test (chi-square $=5.67 ; p=0.225$ )

\begin{tabular}{ccccc}
\hline & Classe 1 & Classe 2 & Classe 3 & Total \\
\hline CTS & $69(79,26)$ & $48(37,94)$ & $11(10,79)$ & 128 \\
CEL & $395(385,16)$ & $174(184,39)$ & $53(52,45)$ & 622 \\
P & $6(5,57)$ & $3(2,67)$ & $0(0,76)$ & 9 \\
Total & 470 & 225 & 64 & 759 \\
\hline
\end{tabular}

\section{CONCLUSÕES}

Infere-se que as espécies do componente arbóreo adulto estão representadas, principalmente, na 
classe de menor tamanho, na qual se encontra a maior parte dos indivíduos amostrados. Isto evidencia o particionamento do perfil vertical da floresta por espécies que apresentam diferentes estratégias de vida, não associadas, no presente trabalho, às guildas de regeneração. Desta forma, os resultados indicam que o potencial regenerativo da floresta em questão é baseado, em parte, na formação de banco de plântulas, que representou uma importante estratégia de regeneração natural da comunidade de espécies avaliada.

\section{AGRADECIMENTOS}

Ao Programa de Bolsas do Fundo de Apoio à Manutenção e ao Desenvolvimento da Educação Superior do Estado de Santa Catarina, pela concessão de bolsa à primeira autora do trabalho. Ao Conselho Nacional de Desenvolvimento Científico e Tecnológico, pela concessão de bolsa de produtividade para o oitavo e o nono autores.

\section{REFERÊNCIAS}

ANGIOSPERM PHYLOGENY GROUP. An update of the Angiosperm Phylogeny Group classification for the orders and families of flowering plants: APG IV. Botanical Journal of the Linnean Society, London, v. 181, n. 1, p. 1-20, 2016.

ARAGÓN, R.; GROOM, M. Invasion by Ligustrum lucidum (Oleaceae) in NW Argentina: early stage charactesistics in different habitat types. Revista de Biología Tropical, San Jose, v. 51, n. 1, p. 59-70, 2003. ASSIS, R. L.; WITTMANN, F. Forest structure and tree species composition of the understory of two central Amazonian várzea forests of contrasting flood heights. Flora, Jena, v. 206, n. 3, p. 251-260, 2011.

BROWN, N. D.; WHITMORE, T. C. Do dipterocarp seedlings really partition tropical rain forest gaps? Philosophical Transactions of the Royal Society of London B: Biological Sciences, Londres, v. 335, n. 1275 , p. $369-378,1992$.

CITADINI-ZANETTE, V. Fitossociologia e aspectos dinâmicos de um remanescente da Mata Atlântica na microbacia do Rio Novo, Orleans, SC. 1995. 236 f. Tese (Doutorado em Ecologia e Recursos Naturais) - Universidade Federal de São Carlos, São Carlos, 1995.

FELFILI, J. M. et al. Cerrado: manual para recuperação de Matas de Galeria. Planaltina: Embrapa Cerrados, $2000.45 \mathrm{p}$.

GAMA, J. R. V.; BOTELHO, S. A.; BENTES-GAMA, M. M. Composição florística e estrutura da regeneração natural de Floresta Secundária de Várzea Baixa no Estuário Amazônico. Revista Árvore, Viçosa, MG, v. 26, n. 5, p. 559-566, 2002.

GEORGE, L. O.; BAZZAZ, F. A. The fern understory as an ecological filter: emergence and establishment of canopy-tree seedlings. Ecology, Washington, v. 80, n. 3, p. 833-845, 1999.

GUIDINI, A. L. et al. Invasão por espécies arbóreas exóticas em remanescentes florestais no Planalto Sul Catarinense. Revista Árvore, Viçosa, MG, v. 38, n. 3, p. 469-478, 2014.

HIGUCHI, P. et al. Florística e estrutura do componente arbóreo e análise ambiental de um fragmento de Floresta Ombrófila Mista Alto-Montana no município de Painel, SC. Ciência Florestal, Santa Maria, v. 23, n. 1, p. 153-164, 2013a.

HIGUCHI, P. et al. Influência do clima e rotas de migratórias de espécies arbóreas sobre o padrão fitogeográfico de florestas na região sul do Brasil. Ciência Florestal, Santa Maria, v. 23, n. 4, p. 539-553, 2013 b.

HIGUCHI, P. et al. Fatores determinantes da regeneração natural em um fragmento de floresta com araucária no Planalto Catarinense. Scientia Forestalis, Piracicaba, v. 43, n. 106, p. 251-259, 2015.

HUMMEL, R. B. et al. Análise preliminar da invasão biológica por Ligustrum lucidum W.T. Aiton em unidade de conservação no Rio Grande do Sul. Caderno de Pesquisa, Série Biologia, Santa Cruz do Sul, v. 26, n. 3, p. $14-26,2014$.

IBGE. Manual técnico da vegetação brasileira. Rio de Janeiro: Fundação Instituto Brasileiro de Geografia e Estatística, 2012. $271 \mathrm{p}$.

INMAN-NARAHARI, F. et al. Seedling recruitment factors in low-diversity Hawaiian wet forest: towards global comparisons among tropical forests. Ecosphere, Whashington, v. 4, n. 2, 2013.

Ci. Fl., v. 28, n. 1, jan.- mar., 2018 
JANZEN, D. H. Herbivores and the Number of Tree Species in Tropical Forests. The American Naturalist, Chicago, v. 104, n. 940, p. 501-528, 1970.

JOHNSON, N. C.; GRAHAM, J-H.; SMITH, F. A. Functioning of mycorrhizal associations along the mutualism-parasitism continuum. New Phytologist, Malden, v. 135, n. 4, p. 575-585, 1997.

KOBE, R. K. Light gradient partitioning among tropical tree species through differential seedling mortality and growth. Ecology, Washington, v. 80, n. 1, p. 187-201, 1999.

LARPKERN, P.; MOE, S. R.; TOTLAND, Ø. Bamboo dominance reduces tree regeneration in a disturbed tropical forest. Oecologia, Nova York, v. 165, n. 1, p. 161-168, 2011.

LOREGIAN, A. C. et al. Padrões espaciais e ecológicos de espécies arbóreas refletem a estrutura em mosaicos de uma floresta subtropical. Acta Botanica Brasilica, Feira de Santana, v. 26, n. 3, p. 593-606, 2012.

OKSANEN, J. et al. Vegan: community ecology package. [2015]. Disponível em: <http://cran.r-project. org/package=vegan $>$ Acesso em: 15 mar. 2015.

OLIVEIRA FILHO, A. T. et al. Effects of soils and topography on the distribution of tree species in a tropical riverine forest in south-eastern Brazil. Journal of Tropical Ecology, Cambridge, v. 10, n. 4, p. 483-508, 1994.

PALMIOTTO, P. A. et al. Soil-related habitat specialization in dipterocarp rain forest tree species in Borneo. Journal of Ecology, Malden, v. 92, n. 4, p. 609-623, 2004.

POORTER, L. Are species adapted to their regeneration niche, adult niche, or both? The American Naturalist, Chicago, v. 169, n. 4, p. 433-442, 2007.

R DEVELOPMENT CORE TEAM. R: a language and environment for statistical computing. Vienna: R Foundation for Statistical Computing, 2014. Disponível em: <http://www.r-project.org>. Acesso em: 15 mar. 2015.

SALLES, J. C.; SCHIAVINI, I. Estrutura e composição do estrato de regeneração em um fragmento florestal urbano: implicações para a dinâmica e a conservação da comunidade arbórea. Acta Botanica Brasilica, Feira de Santana, v. 21, n. 1, p. 223-233, 2007.

SANTOS, K. F. et al. Regeneração natural do componente arbóreo após a mortalidade de um maciço de taquara em um fragmento de Floresta Ombrófila Mista em Lages, SC. Ciência Florestal, Santa Maria, v. 25, n. 1, p. 107-117, 2015.

SCCOTI, M. S. V. et al. Mecanismos de regeneração natural em remanescente de Floresta Estacional Decidual. Ciência Florestal, Santa Maria, v. 21, n. 3, p. 459-472, 2011.

SCHILLING, A. C.; BATISTA, J. L. F. Curva de acumulação de espécies e suficiência amostral em florestas tropicais. Revista Brasileira de Botância, São Paulo, v. 31, n. 1, p. 179-187, 2008.

SCHORN, L. A.; GALVÃO, F. Dinâmica da regeneração natural em três estágios sucessionais de uma floresta ombrófila densa em Blumenau, SC. Floresta, Curitiba, v. 36, n. 1, p. 59-74, 2006.

SILVA, W. C. et al. Estudo da regeneração natural de espécies arbóreas em fragmento Floresta Ombrófila Densa, Mata das Galinhas, no Município de Catende, Zona da Mata Sul de Pernambuco. Ciência Florestal, Santa Maria, v. 17, n. 4, p. 321-331, 2007.

SOUZA, A. F. Ecological interpretation of multiple population size structures in trees: the case of Araucaria angustifolia in South America. Austral Ecology, Malden, v. 32, n. 5, p. 524-533, 2007. SOUZA, K. et al. Estrutura e estratégias de dispersão do componente arbóreo de uma floresta subtropical ao longo de uma topossequência no Alto-Uruguai. Scientia Forestalis, Piracicaba, v. 43, n. 106, p. 321-332, 2015.

SWAINE, M. D.; WHITMORE, T. C. On the definition of ecological species groups in tropical rain forest. Vegetatio, Dordrecht, v. 75, n. 1-2, p. 81-86, 1988.

VIBRANS, A. C. et al. Inventário Florístico Florestal de Santa Catarina: Floresta Estacional Decidual. Blumenau: Edifurb, 2012a. v. 2.336 p.

VIBRANS, A. C. et al. Inventário Florístico Florestal de Santa Catarina: Floresta Ombrófila Mista. Blumenau: Edifurb, 2012b. v. 3. 440 p. 
VOLPATO, M. M. L. Regeneração natural em uma floresta secundária no domínio de Mata Atlântica: uma análise fitossociológica. 1994. 123 f. Dissertação (Mestrado em Ciência Florestal) - Universidade Federal de Viçosa, Viçosa, MG, 1994.

WEBB, C. O.; GILBERT, G. S.; DONOGHUE, M. J. Phylodiversity-dependent seedling mortality, size structure, and disease in a Bornean rain forest. Ecology, Washington, v. 87, n. sp7, p. S123-S131, 2006. 